\title{
المنهج التاريخي قيمته وآلية توظيفه
}

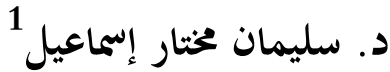 \\ كلية الآداب- جامعة مصراتة
}

https://doi.org/10.36602/faj.2018.n12.04

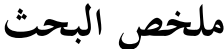

لأن الإنسان انعكاسُ بيئته ونتِاج مجتمعه، فلا شك في كونه يتأثر بهما، ولعل هذا الأثر يظهر جليّا لدى الأديب أكثر من غيره، فهو حين يعبر عن أفكاره فيكتبها وينشرها، فإنه يترك بصمة تدل على أنه ابن ذلك الزمان، أو المكان، أو المنهج. تتعدد المناهج النقدية، ويعد المنهج التاريخي من أول المناهج التي عنيت بالبحث والدراسة بغية دراسة تطور الفكر واللغة، وبهدف الاستفادة من حقائق التاريخ، ونظرياته، وقوانينه في دراسة الأدب ونقده، في هذه الدراسة يهدف الباحث إلى بيان معنى هذا المنهج، وشرح حقيقته، فيتتبع دلالة الكلمة، ويبين أهمية المنهج التاريخي في جوانب العلم المختلفة، كما يفرّق بين تاريخ الأدب والتاريخ العام، ويعرّف الباحث بالمنهج التاريخي

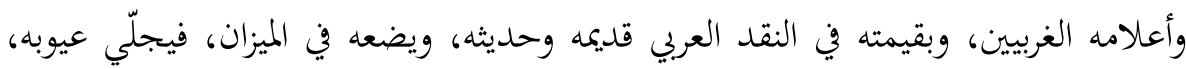
وما أخذ عليه من مآخذ، ثم ينتهي الباحث إلى عدد من النتائج والمستخلصات التي تؤكد أنه- وعلى الرغم من أهميته- فإنه ليس للمنهج التاريخي أن يستقل بنفسه، فلابد أن يسانده المنهج الفني؛ لأن التذوق والحكم ودراسة الخصائص الفنية ضرورة في كل مرحلة. الكلمات المفتاحية: المنهج التاريخي، المناهج الأدبية، النتد العربي. 


\section{Abestract}

The culture of any society influences thoughts, behaviors, trends, tastes and it can influence literature and poetry as well. Also, we are a reflection of our societies. Writers can show that effect in a clearer way more than others as they can reflect this impact by expressing their ideas through writing them down. That gift of writing gives the writers the ability to mirror their backgrounds, then their writings could be considered as a fingerprint which indicates their time, place and method.

There are many critical methodologies, and the historical approach is one of the first approaches that has received the attention of scholars in order to study the evolution of thought and language .In this study, the researcher aims at presenting and explaining the meaning of this historical approach by defining it and showing its importance in different scientific aspects. The researcher also tries to distinguish between the history of literature and the general history, showing the value of the historical approach in the old and modern Arabic criticism and putting it in the balance to illustrate its disadvantages and problems. At the end of the study, the researcher lists several results and confirms that the historical approach is important, but it cannot rely on itself. So, it needs and must be supported by the technical approach as the technical characteristics are necessary at every stage

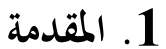

إذا كانت وظيفة النقد الأدبي وغايته تتلخص في تقويم العمل الأدبي من الناحية الفنية والموضوعية، وقيمه التعبيرية والثعورية، وتعيين مكانه في خط سير الأدب، وقياس مدى تأثره بالمحيط و تأثيره فيه، وكشف العوامل النفسية التي اشتركت في تكوينه، والعوامل الخارجية التي أسهمت في تشكيله، وتصوير سمات صاحب العمل الأدبي من خلال أعماله، فما هو المنهج الذي يمكن اتباعه لتحقيق هذه الغايات؟ 
إن المناهج النقدية كثيرة ومتنوعة، فمنذ بداية القرن التاسع عشر تقدمت الدراسات التاريخية، وازدهرت العلوم الطبيعية، فحاول الباحثون والنقاد تبعا لذلك الاستفادة من حقائق التاريخ، ونظريات تلك العلوم وقوانينها في دراسة الأدب ونقده... فكان المنهج التاريخي - موضوع هذا البحث- من أول المناهج النقدية التي طبقت على الأدب يهدف هذا البحث إلى عرض وبيان معنى المنهج، وتتبع دلالة هذه الكلمة في معاجم اللغة، وفي اصطلاح أهل الاختصاص، كما يقف عند الفرق بين تاريخ الأدب والتاريخ العام، ثم يبين أهمية المنهج وضرورته في أي جانب من جوانب العلم والمعرفة، وفي كل دراسة يرغب الباحث خوض غمارها، ثم يعرض البحث لظهور المنهج التاريخي وأعلامه الغربيين، ومعرفته في النقد العربي قديمه وحديثه، وأختم ببيان عيوبه، وما أخذ عليه، وخاتمة بأهم النتائج والمستخلصات، وثبت بالمصادر والمراجع.

\section{2 - 2 المنهج والإجراءات}

يهدف هذا البحث إلى عرض وبيان معنى المنهج، وتتبع دلالة هذه الكلمة في معاجم اللغة، وفي اصطلاح أهل الاختصاص، كما يقف عند الفرق بين تاريخ الأدب والتاريخ العام، ثم يبين أهمية المنهج وضرورته في أي جانب من جوانب العلم والمعرفة، ويف كل دراسة يرغب الباحث خوض غمارها، تم يعرض البحث لظهور المنهج التاريخي وأعلامه الغربيين، ومعرفته في النقد العربي قديمه وحديثه، وأختم ببيان عيوبه وما أخذ عليه، وخاتمة بأهم النتائج والمستخلصات، وثبت بالمصادر والمراجع. 


\section{3. المنهج التاريخي مفهومه ومعناه \\ 3. 1 المنهج في اللغة والاصطلاح}

يرى الناظر في معاجم اللغة أن معنى (المنهج) أو (المنهاج) هو الطريق الواضح، و(النهج)- بتسكين الهاء- هو الطريق المستقيم، ويف القرآن الكريم شرعة ومنهاجاهيم. (سورة المائدة، الآية: 48)، ( ابن منظور، مادة: فج)، وأضاف (المعجم الوسيط( دلالة محدثة لكلمة (المنهج)، فقال: هو الخطة المرسومة، وقال: ومنه منهاج الدراسة، ومنهاج التعليم ونهوها، وفي (معجم النقد العربي القديم) يشير أحمد مطلوب إلى أن المنهج هو الطريق والاسلوب، وقد استعمل حاتم القرطاجني مصطلح المنهاج في بعض أقسام كتابه (منهاج البلغاء وسراج الأدباء) يريد به الباب، وكان قد قسم كتابه إلى أربعة أبواب، أو أقسام، فسمى كل قسم منهجا، وقسم المنهج إلى فصول. أما المعنى العام للمنهج فهو الأسلوب الذي يقود إلى هدف معين في البحث والتأليف والسلوك، (مجلة

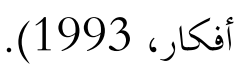

كما يُعرَّف المنهج بأنه: وسيلة محددة توصل إلى غاية معينة، أما المنهج العلمي فهو خطة منظمة لعدة عمليات ذهنية، أو حسية، بغية الوصول إلى كشف حقيقة، أو البرهنة عليها. (وهبة، والمهندس، 1984، 393).

3. 2 أهمية المنهج

يشكل المنهج- في ظل أي قراءة نقدية حديثة أو قديمة- خطوة بالغة الأهمية والقيمة، فهو يضبط خُطى القراءة، ويحدد مسارها، والمنهج هو الذي يتوقف على استقامته أو انحرافه، استقامةُ النتائج التي تفضي إليها القراءة أو انحرافها. 
المنهج هو الذي يترتب على وضوحه ودقة آلياته استشراف آفاق النص، واستشفاف جوانبه، واستجلاء مكنوناته أو مكوناته، ويترتب على ذلك الإقبال على النص بك أو النفور منه، فالمنهج ضرورة لازمة لأي قراءة أو دراسة نصية، بل هو شرطها الحيوي الأول، وإن قراءة من غير منهج واضح لا ينتظر منها نتائج يعتمد عليها في أغلب الأحيان، وإن وجدت فغالبا ما تكون تلك النتائج مشوهة ومحرفة. (المومني، 1999، 96).

فالمنهج هو قائد الأفكار وعاصمها من الزلل، ليس هذا فحسب، بل هو قائد أخلاقي؛ لأن روح العلم روح أخلاقية، وكما يخشى على الفرد الذي يزاول الحياة العملية من الانخراف عن مبادئ الشرف، فكذلك يخشى من الخطر نفسه على من يزاول أعمال الفكر، بل ربما كان الخطر هنا أعظم وأشد؛ لأن وقائع الحياة قد ينبعث منها الجزاء، أما الفكر فإنه وإن يكن ضرر الانحراف فيه أكبر وخطره أوسع انتشارا، إلا أن الجزاء فيه قد لا يكون سريعا، ولا فعلا، ولا أكيدا؛ لأنه لا يعدو أن يكون فقد المؤلف ثقة القراء، وتلك مسألة هروب.( لانسون، 12،. 13 )

ولعل أول منهج وضع للبحث العلمي وطرق الاستدلال هو منهج أرسطو، الذي سماه باسم (المنطق)، وتحدث فيه عن الكليات الخمس (الجنس، والنوع، والفصل، والخاصة، والعرض)، وقد أسلم الأوربيون أنفسهم لمنطق أرسطو في العصور الوسطى، معتقدين بأنه كافٍ في استنباط القوانين العامة كلها، حتى كان فرنسيس بيكون الذي أسس المنطق الحديث، ومن بعده ديكارت، الذي هاجم المنهج الأرسطي، ووضع للعلوم كلها منهجا واحدا، صوره في بحثه (مقال في المنهج)، ورأى أن يضع مكان قواعد المنطق الأرسطي القديم شديدِ التعقيد أربعَ قواعد، تختصر المنهج السديد لكل البحوث النظرية، هي: قاعدة اليقين، والتحليل، والتركيب، والاستقراء التام، ومن حينها كثر القائلون بأن المنطق الأرسطي 
قد انتهى زمنه، وأن الدراسات يجب أن تعتمد على رصد الظواهر وتتبعها، وأنه لا بد من الجمع بين التفكير النظري، والملاحظة والتجربة (ضيف، 1972 ـ 85). 3. 3 التاريخ العام وتاريخ الأدب

يُعرَّف التاريخ العام بأنه: جملة الأحداث التي يمر بها كائن ما، وتصدق على الفرد والمجتمع، كما تصدق على الظواهر الطبيعية والإنسانية، و(التأريخ)- بالهمز - هو تسجيل هذه الأحداث.

أما تاريخ الأدب، فهو تطبيق مناهج التاريخ على وصف الأدب في عصر أو عصور متتالية عند شعب واحد، أو شعوب مختلفة، فعلى المؤرخ الأدبي أن يحدد العصور التي يريد دراستها، مبينا أهم ابتحاتها، ومحاولا الربط بين إنتاج أدبائها، وبين الحدث الأدبي الذي يريد دراسته، والأحداث التاريخية العامة، سياسية كانت أم اجتماعية، وعليه أيضا أن يصف الأدب لا كظاهرة ثابتة، وإنما كظاهرة تتغير وتتطور مع مرور الزمن، نتيجة لرد فعل، أو تأثر، وتفاعل بين عناصر أدبية مختلفة بين العصور. (وهبة، والمهندس، 1984، 84) فتاريخ الأدب جزء من تاريخ الحضارة الذي يجوي تيارات الأفكار، والمشاعر الممتدة من الأحداث السياسية، والاجتماعية، والمركزة على النظم، فالتاريخ الأدبي يهاول أن يصل إلى الوقائع العامة، ويميز الوقائع الدالة، ومن ثم يكشف العلاقة بين الوقائع العامة والوقائع

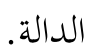

إن موضوع التاريخ هو الماضي الذي لم يبقَ منه إلا أمارات، أو أنقاض، بواسطتها يعاد بعثه، أما تاريخ الأدب فهو ماضٍ، ولكنه ماضٍ باقٍ، فهو من الماضي والحاضر معا. 
إن مادة المؤرخ للأدب هي المؤلفات التي أمامه، التي تؤثر فيه، كما كانت تؤثر في

$$
\text { أول جمهور عرفها، (لانسون، 1982، 26.، 31). }
$$

ومن المناهج العلمية المتبعة في الوصول إلى الحقائق التي يراد كشفها، المنهج التاريخي، فهو من المناهج الخارجية، شأنه شأنُ المنهج النفسي والاجتماعي.

\section{4 3 ظهور المناهج الأدبية}

بدأ البحث الأدبي يتجه ابتحاها منهجيا منذ البعث العلمي في بداية القرن الثامن عشر، فقد ازدهرت الحياة العلمية، وبدأت تصبغ الفكر كله بصبغة علمية منهجية، وكانت الدراسات التاريخية قد تقدمت، وبدأ الباحثون في الأدب ونقاده على السواء يستفيدون من مناهجها، ويحاولون تطبيقها على الأدب والنقد. (ظلام، 1996، 13).

ويعد المنهج التاريخي أول المناهج النقدية ظهورا في العصر الحديث، فقد ارتبط بالفكر الإنساني، وبالتطور الأساسي له، وانتقاله من العصور الوسطى إلى العصور الحديثة، ( فضل، 1997، 24، وهويدي، 1996 ،71)، فقد دخلت أوربا مع القرن التاسع عشر- كما ذكرنا- مرحلة فضة علمية، حين تطورت العلوم الطبيعية التجريبية تطورا مذهلا، كانت له نتائجه على واقع المجتمع، مما دفع النقاد إلى أن يضعوا للأدب قوانين كقوانين العلوم الطبيعية، أسموها بالتاريخ الطبيعي للأدب، فطبقوا على الأدب والأدباء منهج الطبيعيين في تصنيف النبات والحيوان، ورتبوا الأدباء طبقات، وصنفوهم فصائل بحسب خصائصهم الأدبية، وطبق عليهم بعض الدارسين-كما سيأتي- قوانين الجنس والبيئة والزمان، وطبق آخرون على الأدب نظرية دارون في النشوء والارتقاء، أو تطور الكائنات. (ظلام، 1996، 13). 
لقد تبلور المنهج التاريخي داخل المدرسة الرومانسية وانبثق عنها، فالرومانسية هي التي بلورت وعي الإنسان بالزمن، وتصوره للتاريخ، ووضوح فكرة التسلسل، والتطور والارتقاء، والقضاء على فكرة الدورات الزمانية، والحركة الانتكاسية للزمن والتاريخ، التي كانت تضع العصور الذهبية في الماضي، وتنظر إلى الحاضر باعتباره تحللا واهيارا، وتصدعا وتدهورا، هذه هي الفكرة التي كانت سائدة، وهي فكرة الكلاسيكية، فجاءت الرومانسية وعكست هذا المفهوم بشكل أساسي، فكان تصورها لمسيرة الإنسان في الزمن طبقا لقوانين النشوء والارتقاء، والتطور والانتقال، من المرحلة البدائية إلى المرحلة الأكثر تقدما. التطور التاريخي عكس النظرية الكلاسيكية التي كانت ترى أن الأدب محاكاة للأقدمين، وأن الأقدمين يمثلون النموذج الأرقى في مجال التطور التاريخي، فجاءت الرومانسية ووضعتهم في موضعهم الطبيعي في سلم التطور البشري. (فضل، 1997، 24،

\section{5 أهم أعلام المنهج التاريخي}

يقف الدارس على ثلاثة من النقاد الغربيين، يُعتقد أفمم أهم أعلام المدرسة التاريخية، وهم: 1869 ـ سانت بوف 1804 ـ 1869

سانت بوف هو أول ناقد سعى إلى تأسيس تاريخ طبيعي للأدب، فدرس أدباء

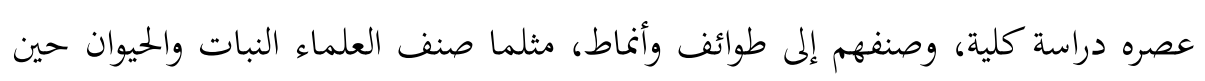
حددوا فصائلها.

كان بوف يؤمن بالعلاقة التي تربط بين الأديب وأدبه؛ فاهتم بدراسة الشخصية اهتماما بالغا، ودرس أذواق شخصيات الأدباء دراسة عضوية ونفسية واجتماعية، كما 
تدرس الثمرة في شجرتا لتتبين خصائصها، وقسم الأدباء إلى طبقات وفصائل وأنواع بحسب ما بينهم من تشابه، على نحو ما يصنع علماء النبات، فاستحال النقد على يديه إلى علم يمكن أن يسمى بالتاريخ الطبيعي للأدب.

سانت بوف لم يهتم بدراسة النص الأدبي ذاته، وكانت لديه قدرة على تتبع الأدباء

والتجسس على حياتم الخاصة؛ فاستقصى الظاهرة المادية والعقلية والأخلاقية، حتى إنه كان لا يتورع في سبيل معرفة ما كان يسميه (بوعاء الكاتب)، فهتك من أجل ذلك الأسرار الخاصة والحياة الخاصة للأدباء، وأدى ذلك إلى امتعاض معاصريه، أما فيما يخص دراسته للأدباء القدامى فقد اعترف سانت بوف بأنه من العسير أن نتتبع حياتم تتبعا دقيقا؛ لأن ما وصلنا عنهم صورة ناقصة. (ظلام، 1996، 21 ـ 22).

فنجد سانت بوف قد أهمل الجوانب المميزة لشخصيات الأدباء، وهي الجوانب التي تجعل لكل واحد منهم كيانا مستقلا، ولكل واحد منهم أصالته، وملامحه، وطباعه الخاصة التي تميز بها وتفرد عن نظرائه في عصره وبيئته، فالشخص الواحد لا يمكن أن يتكرر، فلا بد من وجود اختلاف، فأسقط بوف أهم ما يمتاز به الأدباء من فردية وذاتية. (ضيف،

\section{2. هيبوليت تين: 1828 ـ 1893}

هو ناقد فرنسي حمل لواء الدعوة إلى المنهج التاريخي الجديد بعد أستاذه سانت بوف، وإن كان أكثر تحمسا لمذا المنهج من أستاذه، فهو يرى أن الأديب إنسان سام، ينتج الأشعار والفلسفة مثلما تنتج دودة القز خيوط الحرير، فأسقط الفردية الأدبية إسقاطا تاما، ونفى أن تكون هناك خصائص ادبية يتميز بها الأديب، إنما هناك خصائص جماعية 
تجمع بينه وبين دباء أمته، (ضيف، 87)، جاعلا هذه القوانين قوانين حتمية، وهي لا تخرج عن ثلاثة عوامل: 1 ـ الجنس. 2 ـ البيئة. 3 ـ العصر.

فهو يرى أن أدباء كل أمة يخضعون لهذه القوانين الثلاثة خضوعا جبريا ملزما، فلكل جنس خصائصه ولكل بيئة مناخها وجغرافيتها الخاصة، ولكل زمان وكل عصر أحداثه، وظروفه السياسية، والاقتصادية، والثقافية، والدينية.

فإذا توافرت هذه القوانين لأمة من الأمم أنتجت أدبا؛ لتوافر دواعيه وعوامله، وهو هذا يتجاهل العبقرية والموهبة الفذة؛ لأن العبقرية لا يمكن أن تخضع لظروف، ولا تحدها قوانين. ( ظلام 1996، 23).

ويقصد تين بالجنس السلالة المتمثلة في مجموعة الصفات الموروثة في الأمة، فلكل أمة خصائصها، وهذه فكرة نجدها حتى لدى غيره، كالجاحظ في حديثه عن الأجناس، وابن خلدون في مقدمته عند حديثه عن الجنس العربي وخصائصه، وأثر ذلك في حياته السياسية، كما نجد ذلك عند رنان ـ 1823 ـ 1892، حيث ذهب إلى أن الأمم الآرية تمتاز عن الأمم السامية بفلسفتها وشعرائها، وفنوها وآدابها الرفيعة، لكن هذه الفكرة ذهبت اليوم أدراج الرياح ولم يعد لها اثر، فهي فكرة خاطئة؛ لأن الجنس إنما هو أناس سكنوا إقليما واحدا، وأخذوا يعيشون فيه معا، حتى تشاهت عاداتم وتقاليدهم ومعارفهم، فأفضلية الجنس مقولة خاطئة وحاول الاستعمار الأبيض ترويجها، فادعى أن البياض رمز تقدم بخلاف السواد. (ضيف، 1972، 89 ).

أما البيئة: فيعني بها المكان والمميزات الجغرافية له، الذي ينشأ فيه أفراد الأمة نشوءا يعدهم لممارسة حياة مشتركة في العادات والأخلاق والروح الاجتماعية. 
أما الزمان: فيمثل واقع التيارات السياسية التي تسود في حقبة زمنية ما، وكذلك الظروف الاقتصادية والاجتماعية، والعوامل الثقافية والدينية، التي يجيا الأديب في ظلها وينشئ أدبه. ( هويدي، 1996، 75).

لكن تين تدارك هذه الفكرة عندما أشاد بالعبقرية الشخصية لدى شكسبير في مقدمة كتابه عن الأدب الإنجليزي. (فضل، 1997 ، 34 ـ 35). 3 ـ فرديناند برونتيير . 1849 ـ 1906

أضاف هذا الأديب والمفكر الفرنسي محاولة ثالثة تقوم على نظرية دارون في النشوء والارتقاء، وكان قد سبقه إلى نقلها من العضويات إلى المعنويات (سبنسر)، وطبقها على الأخلاق والاجتماع، فحاول برونتيير أن ينقلها إلى الأدب ليصل إلى كيفية نشوء الأنواع الأدبية، وكيف نمت وتطورت من عصر إلى عصر، وقد توصل إلى نتيجة أن الشعر الغنائي الرومانسي في القرن التاسع عشر، تطورَ عن الوعظ الديني الذي شاع في فرنسا في القرن السابع عشر (ظلام، 1996، 23)، غير أننا نجد موضوع استخدام عبارة النشوء والارتقاء، والتدليل بها على تطور الخطابة الدينية مسألة فيها نظر، فـ(لانسون) يقول: وهذه عبارة لا معنى لها إلا عند من يعرفون الوقائع، أما عند أولئك الذين يجهلوها فإن معناها خطأ؛ وذلك لأنه ليس في الوقائع ذاتا ما يدل على تطور نوع أدبي إلى آخر، وإنما هو المذهب الذي يرى ذلك، بحيث يكون من الخير أن نسقط هذا الاصطلاح العلمي، ونقول في لغة جميع الناس: "إن الشعر الغنائي في القرن التاسع عشر، قد اتخذ مادة له تلك المشاعر التي لم يكن يعبر عنها في فرنسا خلال القرنين السابع عشر والثامن عشر، إلا بواسطة الخطابة الدينية"، وهذه العبارة لا شك أهما أقل إشراقا من السابقة، ولكنها أوضح

$$
\text { وأصدق. (لانسون، 1982، 49). }
$$


إن نظرية التطور مع صحتها ينبغي أن لا نبالغ فيها كما بالغ (برونتيير)، فتصور أن نوعا أدبيا قد يفنى في نوع أدبي آخر، أو يتحول إليه بحيث يتلاشى فيه على نحو ما تلاشى على رأيه وعظ الوعاظ الفرنسيين في القرن السابع عشر في شعر الرومانسيين في القرن التاسع عشر عند "فيكتور هيجو"، وكان الأولى به أن يقول: إن المشاعر التي كان يشبعها الوعظ الديني في القرن السابع عشر أصبح يشبعها الشعر الغنائي الرومانسي بعد قرنين من

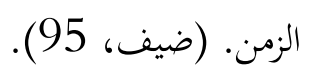

\section{6 التاريخي المنهج في النقد الأدبي عند العرب}

كان النقد العربي عند العربي في بدايته فطريا، يعتمد على اللمحة الذوقية، ولا يعلل، وإن علل فإنه يعلل تعليلا عاما، ولا يفصل، ولا يتجه إلى أصول نقدية، أو يمدد إطارا يتحرك من خلاله، وكان ذلك من آثار الحياة الفكرية والاجتماعية الضيقة والبسيطة في العصرين الجاهلي والإسلامي، ولكن - وبعد أن تطور مفهوم الحياة في العصر العباسي، وازدهرت الثقافة- بدأ النقد يتجه إلى تحديد منهج، وتكوين فلسفة خاصة به، وإرساء قواعد عربية في النقد والبحث. (ظلام، 1996، 28).

وأول من حاول ذلك ابن سلام الجمحي في طبقات الشعراء، فقد سار فيه على ضوء المنهج التاريخي، فخصص في طبقاته مباحث لشعراء القرى العربية، وشعراء المدينة، وشعراء مكة، وشعراء اليهود، ففي هذا التخصيص دليل على معرفته، وتسليمه بأثر البيئة في كل طائفة، وأنه كان يلحظ أن لكل طائفة خصائص مستقلة تميزها، ففي تقسيمه هذا كان يراعي الزمان والمكان. (هويدي، 1996، 75).

ومما يمكن رده إلى عموم الرؤية التاريخية ما ذهب إليه الأصمعي من أثر المتغيرات الاجتماعية والعقدية في شعر حسان بن ثابت في عبارته النقدية المشهورة "الشعر نكد بابه 
الشر، فإذا دخل في الخير لان وضعف"، ثم استدل على صحة قوله بشعر حسان، فقال: "هذا حسان فحل من فحول الشعر في الجاهلية، فلما جاء الإسلام لانَ شعره"، فالأصمعي يرى أن شعر حسان ضعف بعد الإسلام، وقد شهد له الناس في الجاهلية بالفحولة، وما ذلك إلا للتحول الذي طرأ على حياته ومحيطه الاجتماعي والثقافي والفكري، بعد بزوغ فجر الإسلام. (محمد عثمان، 1984، 95 ـ 98). وقد حاول القاضي الجرجاني الربط بين أحوال البداوة والتمدن الاجتماعي والصياغة الأدبية وعادات الناس واخلاقهم، وفي هذا دليل على معرفته بالمنهج التاريخي، وإحساسه بالعوامل المؤثرة في الأدب. (هويدي، 1996، 74 ـ 75). وفي العصر الحديث سار النقد العربي مع النقد الغربي، فأخذ بعض النقاد العرب بالمنهج التاريخي، و تأثروا به، فكتب العقاد (شعراء مصر وبيئاتم في الجيل الماضي)، وكتب طه حسين (مع المتنبي)، (وذكرى أبي العلاء)، و(حديث الأربعاء)، وفي هذه الكتب درسوا البيئات التي عاش فيها الأديب، وأنشأ شعره، والظروف السياسية والاقتصادية، التي أثرت فيه. (ظلام، 1996، وهويدي، 1996). 3. 7 عيوب المنهج التاريخي

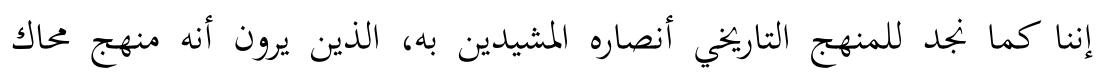
لقوانين العلم وآليات ملاحظته وفحصه ودراسته، فكذلك له خصوم تتبعوه وبينوا عيوبه،

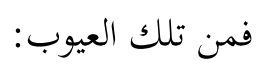
الربط الحتمي بين الأدب والسياسة، والوقوف عند حيوات الملوك والأمراء، وجعل حياة الأدباء مرتبطة بهم ارتباط الظل بالشاخص. يساوي هذا المنهج بين النصوص جميعا جيدها ورديئها؛ لأها جميعا تعكس ظروف العصر وطوابعه. 
يؤدي هذا المنهج إلى جفاف الذوق، والانتقال بالأدب إلى الدرس التاريخي الجامد

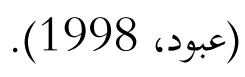

الاستقراء الناقص الذي يؤدي دائما إلى خطأ في الحكم؛ لاعتماده على الحوادث البارزة والظواهر الفذة التي لا تمثل سير الحياة الطبيعية، فألمع الحوادث وأبرز الظواهر ليست أكثر دلالة من الحوادث المهمة والظواهر الصغير.

الأحكام الجازمة التي يصدرها في حوادث قديمة ومسائل تاريخية ليست لدينا جميع مستنداتا، فالظن والترجيح وترك الباب مفتوحا لما يجد كشفه أسلم من الجزم والقطع. التعميم العلمي الذي أوقعهم فيه تطبيق نظرية دارون والنشوء والارتقاء، ولم ينتبه إلى

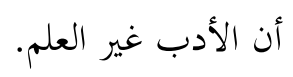

عدم فسح مجال للعبقريات الشخصية، واعتبارها من آثار البيئة والظروف المحيطة

$$
\begin{aligned}
& \text { بالمبدع. (قطب، 1966). } \\
& \text { 5. الاستنتاجات }
\end{aligned}
$$

يؤكد هذا البحث أن الإنسان ابن بيئته، وأن الأديب لابد أن يتأثر بالظروف المحيطة به، وأن للمنهج التاريخي قيمة كبيرة وأهمية بالغة، فهو يعيننا على دراسة تطور الفكر واللغة، إذا ما قارنا مثلا بين شاعرين من بيئة واحدة أو في عصرين مختلفين. كما أن المنهج التاريخي جيد في دراسة الأدب كمنهج خارجي، يضيء جوانب النص، ويكشف المعنى العام للأدب، ويبين أنماطه وأشكاله وتحولاته. 
أما دراسة الطبيعة المجازية للأدب، والأسرار الفنية، والانزياحات اللغوية، والمغامرات

التشكيلية، فإن من العبث البحث عن بتحلياها، ودراستها بهذا المنهج الخارجي، الذي لا يتصل بالنصوص الأدبية، ولا يقوى على معالجتها، بل ربما أضعف صلتنا بالنصوص، فنتصور أن النص معادل لعواطف صاحبه، وتعبير عنها، والحقيقة أن العمل الأدبي تأويل للعاطفة، وليس ترجمة لما . إن المنهج التاريخي لا يستقل بنفسه، فلا بد أن يكون معه قسط من المنهج الفني، فالتذوق والحكم ودراسة الخصائص الفنية ضرورة في كل مرحلة، فمن أراد دراسة الأطوار التاريخية لفن من الفنون، فإنه سيتتبعه منذ نشأته، وسيجمع نصوصه من مصادرها، ثم يرتبها ترتيبا تاريخيا قبل دراستها. وأخيرا، فإن أخطر مخاطر المنهج التاريخي إلغاء قيمة الخصائص، والبواعث الشخصية، وإغفال العبقرية، وحسباها من آثار البيئة، والظروف المحيطة بالمبدع، وهو أمر فيه مغالطة كبيرة.

\section{قائمة المراجع}

أولا: المراجع العربية

ضيف، شوقي. (1972). البحث الأدبي طبيعنه أصوله مصادره، ط6، القاهرة: دار المعارف.

ظلام، سعد. (1996)، مناهج البحث الأدبي دراسة تحليلية تطبيقية، ط2، مكتبة هضة الشرق، جامعة القاهرة. عبود، شلتاغ. (1998). مدخل إلى النقد الأدبي الحلديث. عمان: مجدلاوي للنشر. 
عثمان، محمد. (1984). في أدب الإسلام: عصر النبوة والراشدين وبني أمية. بيروت: دار الأوزاعي.

فضل، صلاح.(1997). مناهج النقل المعاصر. القاهرة: دار الآفاق العربة. قطب، سيد. (1966). النقد الأدبي أصوله ومناهجه، ط4. بيروت: الدار العربية للطباعة والنشر والتوزيع.

لانسون، وماييه (1982). منهج البحث في الأدب واللغة. ترجمة: محمد مندور، ط2. بيروت: دار العلم للملايين. ابن منظور (1988). لسان العرب، بيروت: دار الجيل ودار لسان العرب. المومني، قاسم. (1999). في قراءة النص الأببي. بيروت: المؤسسة العربية للدراسات والنشر.

هويدي، صالح. (1996) النقد الأدبي الحلديث قضابياه ومناهجه. الزاوية: جامعة السابع من أبريل، ليبيا.

وهبة، مجد، والمهندس، كامل (1984). معجم المصطلحات العبية في اللغة والأدب،22. بيروت: مكتبة لبنان. مجلة أفكار (1993). العدد 111. ص.ص56- 66. 